\title{
AMPLIANDO A FAMÍLIA DA SAÚDE: AÇÕES DE FISIOTERAPIA NA ATENÇÃO PRIMÁRIA À SAÚDE
}

\author{
EXPANDING THE HEALTH FAMILY: PHYSIOTHERAPY \\ ACTIONS IN PRIMARY HEALTH CARE
}

Aline Barreto de Almeida Nordi (Orcid: 0000-0003-0262-9796) ${ }^{1}$

Giovanni Gurgel Aciole (Orcid: 0000-0002-6400-8293)

\section{Contato}

Giovanni Gurgel Aciole

E-mail: giovanni.aciole@gmail.com

Departamento de Medicina,

Universidade Federal de São Carlos,

São Paulo, Brasil.

\section{RESUMO}

Este artigo reúne subsídios teórico-práticos que alimentem a agenda de debates sobre a inserção da Fisioterapia no Sistema Único de Saúde (SUS), e foi motivado pela proposta do apoio matricial e da estruturação dos Núcleos de Apoio à Saúde da Família em 2008. A partir de revisão bibliográfica sobre o contexto epidemiológico e político brasileiro, os autores consolidaram um rol de ações para o fisioterapeuta agir na Atenção Primária à Saúde. Em conclusão, consideram que a proposta de inserção desse profissional na equipe interdisciplinar seja potente para entrelaçar a Estratégia Saúde da Família, a Fisioterapia e o Apoio Matricial, de modo a ampliar os benefícios das ações e serviços de saúde para a comunidade e a viabilizar a inclusão de outras categorias profissionais no SUS.

Palavras-chave: Fisioterapia; Saúde da família; Atenção à saúde; Serviços de saúde; sistema único de saúde.

\begin{abstract}
This article gathers theoretical-practical subsidies that feed the debate agenda about the insertion of Physiotherapy in the Unified Health System (SUS), and was motivated by the proposal of matrix support and the structuring of the Family Health the Family Health Support Centers in 2008. Based in the bibliographic review on the Brazilian epidemiological and political context, the authors consolidated a list of actions for the physiotherapist to act in Primary Health Care. In conclusion, they consider that the proposal to insert this professional in the interdisciplinary team is potent to intertwine the Family Health Strategy, Physiotherapy and Matrix Support, in order to expand the benefits of health actions and services to the community and to make possible the inclusion of other professional categories in the SUS.

Keywords: Physical therapy specialty; Family health; Health care (Public health); Health services; Unified health system.
\end{abstract}




\section{INTRODUÇÃO}

Os desafios da sociedade brasileira, marcada por profundas desigualdades socioeconômicas, heterogeneidade infraestrutural e profundas transformações demográficas, demandam mudanças na organização do sistema de atenção à saúde, e vêm impondo a necessidade da redefinição do objeto das práticas de saúde e a inclusão de novos saberes e práticas, tanto para a imposição de defrontar as diferenças regionais de ações e serviços quanto para a necessidade de ampliar e transformar as práticas de atenção, afirmando o seu fundamental caráter públi$\mathrm{Co}^{1,2}$. Reforça essa constatação o fato de que, desde 1988, a saúde tenha tido afirmado o paradigma de sua determinação social ${ }^{3,4}$, e destacada como política pública igualitária, centrada como direito de cidadania e orientada pela consolidação do Sistema Único de Saúde (SUS), cujas premissas básicas de integralidade, qualidade, equidade e participação social colocam instigantes desafios para gestores, profissionais de saúde e formuladores de políticas sociais.

Em outras palavras, isso tem significado a construção de práticas de promoção e prevenção em uma perspectiva multidisciplinar; a adoção de uma prática clínica, individual e familiar, que se desenvolva por meio de ações de humanização, acolhimento, produção de vínculo e responsabilização; a produção do cuidado de forma integral e interdisciplinar; o planejamento e gestão desse cuidado com foco no território e na comunidade adstrita; o trabalho em equipe com corresponsabilidade entre profissionais; o estímulo à participação social e envolvimento solidário das famílias; a intersetorialidade das ações; a discriminação positiva de saúde; e a substituição de práticas tradicionais e a adequada articulação entre as atividades clínicas e de saúde coletiva, impulsionando seu caráter multidimensional mediante a incorporação de novos profissionais ${ }^{5}$. Um conjunto de tarefas que pode ser subsumido na reorientação do modelo assistencial para a transformação e mudança das condições de vida e saúde do povo brasileiro $^{11}$.

Nesse texto, refletiremos sobre a integração possível, e desejável, entre as ações dos profissionais de saúde, a Estratégia Saúde da Família (ESF) e o contexto socioepidemiológico em que acontecem, com vistas à ampliação da família da saúde. Realizamos uma breve contextualização das mudanças demográficas e epidemiológicas em curso e uma leitura crítico-reflexiva das propostas que se delineiam com vistas à estruturação e à montagem de equipes de apoio matricial em que está sendo buscada a inclusão das “novas" profissões da saúde, das quais destacaremos a Fisioterapia. 
TRANSIÇÃO EPIDEMIOLÓGICA E DEMOGRÁFICA: $O$ CONTEXTO BRASILEIRO E A FISIOTERAPIA

A transição epidemiológica em curso no Brasil, fruto das mudanças no perfil demográfico e no modo de vida da população, vem promovendo profundas alterações no padrão de morbimortalidade em todas as faixas etárias, e em todo o espaço geográfico do País. Em 1930, cerca de $50 \%$ das mortes no Brasil eram por doenças infecciosas ou parasitárias. As causas externas correspondiam a 3\%; as neoplasias, a 3\%; e as doenças do aparelho circulatório, a 12\%. Em 2015, as doenças infecciosas e parasitárias respondem por $5,2 \%$ das mortes; as violências, por $13,5 \%$; as neoplasias, por $16,2 \%$; e as doenças do aparelho circulatório, por $28,4 \%{ }^{11}$.

As Doenças Crônicas Não Transmissíveis (DCNT) acometem, atualmente, direta e indiretamente, cerca de três quartos da população adulta. No grupo das DCNT, as doenças cardiovasculares constituem-se a primeira causa de óbito em todas as regiões do País. Entre os fatores e comportamentos de risco, são identificados aqueles ligados ao estilo de vida, as exposições no ambiente de trabalho e outros fatores de risco variados que acentuam ou interagem com outros preexistentes. Vários fatores de risco já estão bem definidos, entre eles, a obesidade, o sedentarismo, a ingestão de álcool e o fumo. Associam-se a estes os fatores de ordem psicossocial, decorrentes de tensões ou conflitos vivenciados no ambiente de trabalho e familiar, reconhecidamente geradores de estresse ${ }^{10}$.

No contexto da saúde pública contemporânea, outro grande desafio é o envelhecimento populacional. No Brasil, o número de idosos (acima de 60 anos de idade) passou de 3 milhões em 1960 para 7 milhões em 1975 e 14 milhões em 2002 (um aumento de $500 \%$ em 40 anos); e estima-se que alcançará 32 milhões já no ano que vem ${ }^{12}$. Doenças próprias do envelhecimento ganham maior expressão no conjunto da sociedade. Um dos resultados dessa dinâmica são a demanda crescente por serviços de saúde e a escassez de recursos. O idoso consome mais serviços de saúde, as internações hospitalares são mais frequentes, e o tempo de ocupação do leito é maior quando comparado a outras faixas etárias. Em geral, as doenças dos idosos são crônicas e múltiplas, exigem acompanhamentos constantes, medicação contínua e exames periódicos ${ }^{12}$.

O Acidente Vascular Encefálico (AVE) é a principal causa de internações, mortalidade e deficiências na população brasileira. A incidência do AVE é maior após os 65 anos, havendo um aumento do risco com a idade, dobrando a cada década após os 55 anos. Tendo em vista o rápido e intenso envelhecimento populacional brasileiro, estima-se que o AVE tenha cada vez mais relevância como um problema de saúde pública, até que os investimentos na prevenção dessa doença passem a ser prioridade do sistema de saúde brasileiro. Após a hospitalização inicial, $80 \%$ dos sobreviventes dessa enfermidade retornam à comunidade requerendo cuidados especiais ${ }^{12}$.

Ainda persistem, porém, agravos de natureza infecciosa e parasitária, convivendo com as doenças da modernidade. A hanseníase, por exemplo, permanece como questão relevante. Apesar dos avanços do SUS, a doença continua a produzir mais de 40 mil casos novos/ano no Brasil e mantém importante endemicidade. Houve um acréscimo gradual de casos novos de tuberculose no País, no período de 
2000 a 2003, possivelmente em razão da melhora de detecção e do incremento das ações de vigilância nos últimos dois anos. O Sistema de Informação de Agravos de Notificação (Sinan) do Ministério da Saúde detectou 81.053 casos novos de tuberculose em $2003^{11}$, e esses números seguem crescendo.

A saúde do trabalhador também vem ganhando destaque no cenário de saúde brasileiro. As DCNT representam, em conjunto, causa de incapacidade para o trabalho, inicialmente temporária, podendo levar a situações de invalidez determinando aposentadorias precoces. Isso determina um aumento do volume de gastos pela previdência e um elevado custo social devido à elevada morbidade, a mortes prematuras, muitas destas evitáveis, ou à incapacitação de pessoas em idade produtiva $^{10}$. Hermanova (1987) apud Castro ${ }^{13}$ relata uma taxa de $10 \%$ de prevalência de deficiência física na população mundial. Segundo dados do Instituto Brasileiro de Geografia e Estatística (IBGE) - Censo Demográfico (2000), existem, no Brasil 1.422.223 pessoas com deficiência física ${ }^{14}$.

Entre os problemas mais comuns enfrentados pela população brasileira e mundial e que são, muito provavelmente, subnotificados, podem-se destacar os distúrbios osteomusculares relacionados ao trabalho (DORT), as dores lombares, e diversas outras disfunções musculoesqueléticas. As primeiras podem gerar diferentes graus de incapacidade funcional, e são consideradas um dos mais graves problemas no campo da saúde do trabalhador. As doenças reumáticas podem ser agudas, recorrentes ou crônicas, atingindo pessoas de todas as idades. Como causa frequente de incapacidade, essas são, no seu conjunto, as principais responsáveis pelos custos com a saúde - consultas, medicamentos ou cuidados de reabilitação ${ }^{15}$. Já em relação às dores lombares, em alguma época da vida, de $70 \%$ a $85 \%$ de todas as pessoas sofrerão deste mal. Além disso, a lombalgia é causadora de licenças médicas, elevados custos com saúde, comprometimento da produtividade por alto absenteísmo ocupacional. A prevalência de dor lombar crônica é importante quando se considera a quantidade de limitação das atividades e de demanda por serviço de saúde, geradas por esse problema ${ }^{15}$.

A partir da análise da mudança do perfil epidemiológico da população, verifica-se a necessidade de uma atuação ampla da rede de serviços de saúde, que deve ir além do campo das doenças infecciosas, agindo também nas áreas crônicodegenerativas e traumáticas ${ }^{16}$. Essa parece ter sido a atitude coerente tomada pelo conjunto de atores governamentais do SUS - os gestores das três esferas de governo - que, em 2006, firmaram um Pacto pela Saúde, no qual a atenção à saúde do idoso é umas das seis prioridades (Portarias $n^{\circ} 399$, de 22 de fevereiro de 2006; e n 699, de 30 de março de 2006) ${ }^{11}$.

O Ministério da Saúde, por seu turno, vem dando reforço considerável à incorporação de novos saberes e práticas, por meio da Secretaria Executiva; da Secretaria de Atenção à Saúde e da Secretaria de Gestão do Trabalho e da Educação na Saúde. Entre outras ações, por exemplo, quando propõe a criação dos Núcleos de Saúde Integral ${ }^{7}$, com a inserção das ações de Saúde Mental, Reabilitação e Atividade Física no âmbito da Atenção Básica. Nessa proposta, o fisioterapeuta foi alocado na modalidade Reabilitação, juntamente com o fonoaudiólogo, terapeuta ocupacional e assistente social. 
Em 2008, a Portaria MS no 154 , de 24 de janeiro, estabelece os Núcleos de Apoio à Saúde da Família (Nasf) com o objetivo de ampliar a abrangência, o escopo e a resolutividade das ações da Atenção Primária à Saúde (APS), apoiando a inserção da ESF na rede de serviços e o processo de territorialização e regionalização ${ }^{8}$. São constituídos por profissionais de diferentes áreas de conhecimento, atuando em parceria com os profissionais das equipes de Saúde da Família (EqSF), compartilhando as práticas em saúde nos territórios sob a responsabilidade deste, bem como atuando diretamente no seu apoio e na unidade na qual o Nasf está cadastrado.

Os Nasf deverão atuar sob os pressupostos de equipe matricial (inter)ligada a equipes de referência: as EqSF. Entre as ações de responsabilidade de todos os profissionais que compõem as equipes dos Nasf, mencionamos as seguintes: a) identificar, em conjunto com as equipes e a comunidade, as atividades, as ações e as práticas a serem adotadas em cada uma das áreas cobertas, e o público prioritário a cada uma das ações; b) atuar, de forma integrada e planejada; e desenvolver ações coletivamente, com vistas à intersetorialidade; c) promover a gestão integrada e a participação dos usuários nas decisões; d) avaliar, em conjunto com as equipes de saúde e a comunidade, o desenvolvimento e a implementação das ações, e a medida de seu impacto sobre a situação de saúde, por meio de indicadores previamente estabelecidos; e) elaborar projetos terapêuticos individuais, por meio de discussões periódicas que permitam a apropriação coletiva pelas equipes e pelos Nasf do acompanhamento dos usuários, realizando ações multiprofissionais e transdisciplinares e desenvolvendo a responsabilidade compartilhada.

As ações mais específicas foram assim categorizadas: Ações de Atividade Física/Práticas Corporais; Ações das Práticas Integrativas e Complementares; Ações de Reabilitação; Ações de Alimentação e Nutrição; Ações de Saúde Mental; Ações de Serviço Social; Ações de Saúde da Criança; Ações de Saúde da Mulher; Ações de Assistência Farmacêutica.

Apoio matricial e equipe de referência são, ao mesmo tempo, arranjos organizacionais e uma metodologia para a gestão do trabalho em saúde, objetivando ampliar as possibilidades de realizar clínica ampliada e integração dialógica entre distintas especialidades e profissões. Sua utilização como instrumento concreto e cotidiano pressupõe certo grau de reforma ou de transformação do modo como se organizam e funcionam serviços e sistemas de saúde 9 . A equipe de referência é composta por um conjunto de profissionais considerados encarregados de intervir sobre um mesmo problema de saúde, buscando atingir objetivos comuns e sendo responsáveis pela realização de um conjunto de tarefas. Para que ocorra clara definição da responsabilidade sanitária, é fundamental valer-se da metodologia de adscrição de clientela à equipe de referência. Assim, permite-se a avaliação de risco e vulnerabilidade, identificando-se aqueles casos que mereceriam a elaboração de um projeto terapêutico singular, alteração da avaliação diagnóstica ou dos procedimentos de cuidado 9 . 
O apoio matricial em saúde objetiva assegurar retaguarda assistencial e de suporte técnico-pedagógico às equipes de referência; busca personalizar os sistemas de referência e contrarreferência, ao estimular e facilitar o contato direto entre referência encarregada do caso e especialista de apoio; procura construir e ativar espaço para comunicação ativa e para o compartilhamento de conhecimento entre profissionais de referência e apoiadores. $\mathrm{O}$ apoiador matricial é um profissional que tem um núcleo de conhecimento e um perfil distinto daquele dos profissionais de referência, mas que pode agregar recursos de saber e mesmo contribuir com intervenções que aumentem a capacidade de resolver problemas de saúde da equipe primariamente responsável pelo caso ${ }^{9}$.

As duas propostas, vistas como sinérgicas, têm aberto perspectivas de inclusão de "novas" práticas e saberes profissionais nos territórios de ação da atenção primária, promovendo rupturas na lógica predominante que considera medicina, enfermagem e odontologia. Assim, são convidadas a participar da confraria de saberes e fazeres nesse nível da atenção à saúde profissões como: a nutrição, a fisioterapia, a educação física, a psicologia etc. Porém, quase todas se deparam com lacunas e/ou insuficiências no pensar o fazer nesse campo, suscitando, entre seus profissionais, mais desconfiança que otimismo, mais insegurança que desejo de inserção. Por isso, a intenção dos autores, no caso específico da fisioterapia, foi formular um conjunto de ações de modo a contribuir para a visualização concreta do que fazer na atenção primária para essa profissão, à luz das propostas de inserção pelo apoio matricial e nos Nasf.

\section{A FISIOTERAPIA E A SAÚdE DA FAMÍLIA}

A Fisioterapia pode agregar importantes contribuições nesse novo cenário brasileiro, de forma que a integralidade da atenção à saúde possa ser uma variável concreta nas ações do sistema. A despeito de sua regulamentação já remontar a 50 anos, no entanto, esse saberfazer profissional tem-se apresentado, no cenário das práticas de atenção primária, de modo ainda insidioso e paulatino. Essa insipiência pode ser explicada pelo fato de que - dada a sua concepção como prática, e sua conformação ao modelo assistencial curativo durante esse tempo -, a profissão destinou-se, quase exclusivamente, à atuação destinada à cura de determinadas enfermidades e/ou à reabilitação de sequelas e complicações.

Destaquemos, por exemplo, que, em 1969, por meio do Decreto-Lei $n^{\circ}$ 938, sua regulamentação como profissão propôs a terapia física como seu ato profissional e a capacidade física como seu foco. Nesse devir, destacam-se como lócus de atuação fisioterapêutica o hospital e as clínicas, priorizando, valorizando e restringindo à prática fisioterapêutica reabilitadora ${ }^{5}$. De outro lado, a proposição da Fisioterapia Coletiva, englobando e ampliando a Fisioterapia Reabilitadora, e possibilitando o desenvolvimento da prática fisioterapêutica, tanto no controle de danos quanto no controle de riscos, vem sendo vista como uma estratégia dessa aproximação ${ }^{6}$. Reconheça-se que, em 1987, a Resolução no 80, do Conselho Federal de Fisioterapia, por exemplo, adotou 
o movimento humano como sendo seu objeto de estudos, e seu campo de atuação nos diversos níveis de assistência; e o foco da atenção à saúde sob o conceito de qualidade de vida e bem-estar biopsicossocial. Esta deveria aproximar-se também da promoção de saúde e da nova lógica organizativa dos modelos assistenciais, sem abandonar suas competências à reabilitação ${ }^{5}$.

A formação e a atuação dos profissionais de Fisioterapia ainda se pautam, predominantemente, na prática biológica, fragmentada, curativa e voltada ao setor privado ou aos níveis secundário e terciário da atenção à saúde. Estar na APS pode parecer um lugar atemorizante, conflitante e desafiador para esses profissionais. Algumas experiências nesse sentido já estão acontecendo, mas o papel e as atribuições ainda estão sendo construídos por estes e pelas equipes às quais estão se integrando $^{17}$.

Para inserir-se na APS, a competência do fisioterapeuta precisa ir além da boa técnica. É preciso estar sensível às necessidades e às circunstâncias de vida das famílias envolvidas. Devem-se considerar, além disso, a intervenção dependente da flexibilidade dos atores sociais envolvidos e a compreensão de que as ações específicas de sua profissão são insuficientes para dar conta dos problemas que comprometem a saúde das coletividades, mesmo se trabalhando em uma perspectiva interdisciplinar $^{17,18}$.

Para traçar um panorama geral e elencar possibilidades de atuação do fisioterapeuta na APS, fizemos um levantamento bibliográfico com os seguintes critérios de inclusão: serem documentos brasileiros do período entre 1999 e 2009, para avaliar, em um intervalo de uma dé- cada, a gênese e a implantação da ESF no Brasil; contemplar a Fisioterapia na APS e equipe interdisciplinar; e o texto completo estar disponível. A busca foi realizada nas seguintes fontes: a) bases Lilacs, Medline, SciELO com as palavras-chave (terminologia DeCS/MeSH): fisioterapia e atenção primária à saúde; fisioterapia e atenção básica à saúde; fisioterapia e programa de saúde da família. Com a exclusão dos artigos repetidos, chegou-se ao seguinte conjunto de documentos analisados: a) $\operatorname{artigos}^{6,12,13,18-28}$; b) manuais e portarias do Ministério da Saúde ${ }^{7,8} ;$ c) livros da área ${ }^{29,30-}$ ${ }^{32}$; d) monografias, dissertações e teses do acervo pessoal dos autores ${ }^{5,15,21,22}$.

Para contribuir, qualificar e deixar mais acessível para gestores, profissionais, pesquisadores e estudantes da área sobre as possibilidades de intervenção do Fisioterapeuta na APS, buscamos tornar visível e sistematizado suas atribuições em um nível de atenção em que sua atuação é ainda incipiente. Além disso, verificar a viabilidade e necessidade da sua inserção para intervenção nas necessidades de saúde apresentadas em cada contexto.

Tornar as atribuições do fisioterapeuta na APS visíveis e compiladas é importante no sentido de contribuir, qualificar e deixar mais acessível para gestores, profissionais, pesquisadores e estudantes da área sobre as possibilidades de intervenção desse profissional em um nível de atenção em que sua atuação é ainda incipiente. Além disso, demonstrando que é viável e necessária sua inserção para intervenção nas necessidades de saúde apresentadas em cada contexto.

Com base nos textos, nos artigos e no material coletado na busca, estruturamos a compilação abaixo, segundo áreas de atribuição para o fisioterapeuta. 


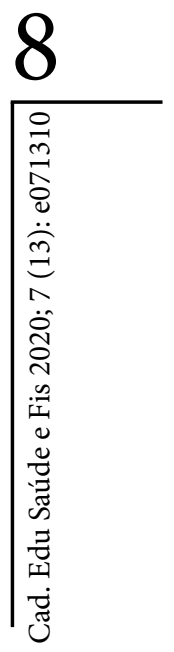

Atribuições gerais:

- $\quad$ Executar ações de assistência integral em todas as fases do ciclo de vida: criança, adolescente, mulher, adulto e idoso, realizando atendimentos individuais ou coletivos, domiciliares, na unidade ou em outros espaços públicos;

- Reconhecer e realizar, multiprofissional, transdisciplinar e intersetorialmente, análises de situação de saúde do território, com identificação dos principais problemas que requerem ações de prevenção e promoção de saúde, bem como de recuperação e reabilitação;

- $\quad$ Realizar visitas domiciliares para orientações, adaptações e acompanhamentos de acordo com a necessidade e complexidade requerida pelo usuário;

- Trabalhar em equipe, realizando discussões e elaborando projetos terapêuticos conjuntos e complementares.

\section{Atividades preventivas e de promoção de} saúde:

- Desenvolver programas de atividades físicas e condicionamento cardiorrespiratório para o obeso, prevenindo com isso a instalação de enfermidades relacionadas com a obesidade;

- Desenvolver ações integradas com os demais equipamentos sociais do território (como escolas, creches, pastorais de saúde, instituições de longa permanência e outros), realizando assessoria, orientação, atividades de promoção à saúde e inclusão, a partir das necessidades identificadas na comunidade;
- Desenvolver ações de promoção e proteção à saúde, incluindo aspectos físicos e da comunicação, como consciência e cuidados com o corpo, postura, autocuidado;

- Desenvolver projetos e ações intersetoriais para a inclusão e melhoria da qualidade de vida das pessoas com deficiência;

- $\quad$ Realizar vigilância dos distúrbios cinesiofuncionais;

- Desenvolver ações de prevenção e promoção das doenças associadas ao trabalho.

\section{Ações em Saúde da criança}

- Desenvolver ações para subsidiar o trabalho das equipes e fortalecer as competências familiares, no que diz respeito ao desenvolvimento infantil;

- Prestar atendimento pediátrico a pacientes portadores de doenças neurológicas com retardo no desenvolvimento neuropsicomotor, más formações congênitas, afecções respiratórias, deformidades posturais;

- Orientar grupo de cuidadores de crianças com asma sobre a doença e sobre os procedimentos a serem adotados antes e durante a crise como manobras de higiene brônquica e das vias aéreas superiores, posturas de drenagem, manuseio e limpeza do nebulizador, consumo de medicamentos e condutas a serem adotadas pelas mães e crianças para manter a tranquilidade e facilitar a tomada de decisões durante a crise. 
Ações em Saúde do adulto

- Orientar atividades físicas em patologias específicas, como a Hipertensão Arterial Sistêmica, Diabetes Melittus, Tuberculose e Hanseníase, principalmente exercícios aeróbicos, exercícios/técnicas respiratórios/relaxamento, a fim de prevenir complicações decorrentes e deformidades que levam às incapacidades;

- Dar suporte às equipes na detecção de agravos e doenças relacionadas ao trabalho, bem como o acompanhamento do itinerário terapêutico.

Ações em Saúde da mulher

- Realizar técnicas de relaxamento, prevenção e analgesia para diminuição e/ou alívio da dor, nas diversas patologias ginecológicas;

- desempenhar condicionamento físico no pré-natal e puerpério, exercícios de relaxamento e orientações de como a gestante deve proceder no pré e no pós-parto para que ela possa retornar às suas atividades normalmente;

- Orientar quanto ao diagnóstico precoce na prevenção de câncer, bem como realizar procedimentos ou técnicas fisioterápicas a fim de evitar as complicações da histerectomia e da mastectomia, incluindo drenagem linfática como forma de tratamento;

- $\quad$ Realizar programas de atividades físicas e psicossociais com o objetivo de aliviar os sintomas do climatério: fase da vida da mulher, na qual cessa seu papel reprodutivo com as correspondentes alterações orgânicas.
Práticas de reabilitação:

- Acolher os usuários que Nordi e Aciole requerem cuidados de reabilitação, realizando orientações, atendimento ou acompanhamento individual, em grupo ou oficinas, de acordo com a necessidade da população e capacidade da unidade;

- Desenvolver ações integradas com as ESF visando à coleta de dados, orientação transmitida à população, detecção precoce e realização de diagnóstico, com levantamento de problemas relacionados com as deficiências, capacitando, orientando, dando suporte às ações dos agentes comunitários de saúde e das necessidades em termos de reabilitação, na área adstrita às ESF;

- Acolher, apoiar e orientar as famílias, principalmente no momento do diagnóstico, para o manejo das situações advindas da deficiência de algum de seus membros, tendo em vista a inclusão e a vida independente;

- Orientar e informar as pessoas com deficiência, cuidadores e familiares sobre manuseio, posicionamento, atividades de vida diária, recursos e tecnologias de atenção para o desempenho funcional frente às características específicas de cada indivíduo;

- Desenvolver ações de Reabilitação Baseada na Comunidade - que pressupõem valorização do potencial da comunidade, concebendo todas as pessoas como agentes do processo de reabilitação e inclusão; 
- Realizar encaminhamento e acompanhamento das indicações, concessões e usos de órteses, próteses e/ou equipamentos auxiliares; encaminhamentos e atendimentos específicos realizados por outro nível de atenção à saúde.

Vigilância dos distúrbios cinesiofuncionais - A vigilância dos distúrbios cinesiofuncionais constitui-se em subárea da vigilância epidemiológica responsável pelo acompanhamento e monitoramento da integridade físico-funcional e dos distúrbios relativos à locomoção humana, devendo acontecer a partir da atenção básica ${ }^{6}$. Para o fisioterapeuta, um desafio da atuação é a falta de informações acerca dos dados epidemiológicos. Em se tratando das demandas encaminhadas pela equipe de saúde, muitas vezes, esta só apresenta dados quanto ao número de acamados ou de casos com $\mathrm{AVE}^{27}$. Os limites dos dados e indicadores na APS podem ser evidenciados pela subnotificação de disfunções musculoesqueléticas, sobretudo quando a Organização Mundial da Saúde promove a "Década do Osso e da Articulação (2000-2010)", sendo esse movimento responsável pela iniciativa de alguns países em pôr em pauta a saúde musculoesquelética da população ${ }^{16}$. De acordo com o atual cenário epidemiológico, algumas condições apresentam maior potencial para limitação da capacidade funcional das populações, a exemplo das patologias relacionadas com o apare- lho cardiocirculatório, com as causas externas e com o aumento da população idosa ${ }^{6}$. Dessa forma, a vigilância e o acompanhamento dos distúrbios cinético-funcionais têm por objetivo fornecerem dados e informações relevantes para as equipes de saúde, tanto na definição do perfil epidemiológico da população como subsidiando as atividades de planejamento e intervenção ${ }^{6}$.

\section{Orientações Posturais}

- A questão da postura deve ser difundida em âmbito coletivo como atitude corporal inerente a uma vida saudável e fator preventivo para diversas doenças. No âmbito da AP, o fisioterapeuta deve atuar preferencialmente com grupos populacionais, com processo de educação e orientação postural construído coletivamen$t e^{6}$. Nesse sentido, dentre as possibilidades de atuação do fisioterapeuta na orientação postural, destaca-se a atuação em grupos de escolares e grupos terapêuticos da postura corporal (grupo de coluna aberto para a comunidade ${ }^{6,26}$. O desenvolvimento de hábitos posturais saudáveis deve começar ainda na fase da infância. A percepção e a conscientização da postura corporal se iniciadas quando da formação dos conceitos iniciais da criança, podem acompanhá-la durante toda a vida, desenvolvendo nos cidadãos consciência da própria postura, instituindo uma cultura de valorização e cuidado com a postura corporal. A educação postural de escolares deve ser uma ati- 
vidade continuada, articulada entre os setores de educação e saúde, com destacada participação dos professores, e que valorize o espaço da escola e o mundo da criança como lócus preferencial de atuação ${ }^{6}$.

- $\quad$ Os grupos de coluna são formados por pessoas diferentes em torno de um objetivo comum, que pode ser: dores de coluna, hipertensão, doença reumática ou neurológica, climatério ou preparação para o parto, DORT, asma ou simplesmente a preocupação com a postura corporal; permitem atendimento em grupo visando minimizar as queixas relacionadas com patologias dessa região, bem como aumentar o conhecimento do corpo e suas funções para promover uma reeducação dos diversos movimentos e posturas; pode proporcionar aos profissionais das equipes (cuidando do cuidador) e à população orientações no que diz respeito à prevenção de algias e más-posturas; e contribuir para o bem-estar de todos os envolvi$\operatorname{dos}^{27}$. A concepção humanizada nesses grupos, onde existe a observação do outro e a troca de experiências, é trabalhada por meio de um maior contato com a respiração consciente, de técnicas de relaxamento e vivências corporais de massagem e automassagem realizadas coletivamente.

\section{Ações em Saúde do idoso:}

- A Fisioterapia pode contribuir para a atenção ampla do idoso no SUS, desenvolvendo interdiscipli- narmente algumas ações, tais como: 11 preservação das funções motoras; minimização ou retardo da dependência funcional; tratamento das alterações motoras e dos sistemas provenientes de doenças e problemas associados; e reabilitação funcional do idoso dentro das suas potencialidades e especificidades; prevenção da imobilidade, promoção das atividades de lazer, estimulo à criatividade e socialização, induzindo a convivência social pelo estímulo a atividade física individual e coletiva ${ }^{27}$. Atuando de forma preventiva e promocional, especialmente nas visitas domiciliares, pode auxiliar na redução das perdas de autonomia produzidas pelo envelhecimento, com ações de estimulação sensório-motora: vigilância de quedas, diminuindo sua possibilidade de ocorrência e consequente hospitalização, pela adoção de ambiente domiciliar seguro e hospitalização; atuação na superação das constantes ameaças ao equilíbrio; na melhora da capacidade funcional e da conscientização de suas limitações; e sugerindo ainda algumas modificações em seus domicílios a fim de conferir maior segurança e independência ${ }^{28}$. As atividades coletivas melhoram a sociabilidade por meio da convivência em grupo, prevenindo assim o isolamento do indivíduo, tão comum nessa faixa etária. Realizar dinâmicas de atividades terapêuticas funcionais em conjunto é estimulante para os idosos, pois o encorajamento e o suporte grupal servem de apoio para a realização delas ${ }^{27,28}$. 


\section{Saúde do cuidador informal}

- Entende-se por cuidador informal a pessoa diretamente ligada e responsável pelo processo de cuidado de um usuário com alguma enfermidade e que necessita da presença constante desse sujeito devido à dependência para o desempenho de diversas atividades. Dessa forma, esse novo público também demanda uma atenção dos profissionais de saúde por estar, com essa atuação, implicado em fatores de risco para sua saúde ou qualidade de vida. O cuidador geralmente é um membro da família ou alguém muito próxima a ela. Muitas vezes, ser cuidador torna-se sua profissão ou sua única atividade, sem, portanto, ter sido capacitado para tal. O senso comum, a responsabilidade e/ ou amor pelo outro fazem com que o aprendizado seja contínuo e na práti$\mathrm{ca}^{29}$. Nesse sentido, a equipe de saúde e o fisioterapeuta, em especial, também dependem muito do cuidador para que suas orientações tenham eficácia e continuidade. Para que o cuidador se sinta seguro para apoiar um membro da sua família, no seu cuidado cotidiano, devem ser direcionados esclarecimentos sobre a patologia, prognóstico e suas implicações físicas; orientações quanto aos cuidados e prevenção de maiores comorbidades; orientações quanto ao manuseio adequado do paciente e quanto aos exercícios e alongamentos de grupos musculares específicos de acordo com as atividades de cuidados realizadas, bem como medidas para alívio de algias musculares; possíveis adaptações ambientais no domicílio e orientações quanto ao uso de órteses e próteses adaptadas às condições domiciliares; orientações quanto à importância da continuidade do tratamento e sua realização diária; e incentivo e valorização do trabalho do cuidador contribuindo para uma melhor compreensão do seu papel sem abdicar de sua vida pessoal ${ }^{29}$. A sobrecarga física e emocional traduzida em problemas físicos, psicológicos ou emocionais, sociais e financeiros relativo às muitas atividades desenvolvidas pelo cuidador, bem como o risco de agravamento das doenças crônicas apresentadas mesmo antes do encargo de cuidar, tem sido alvo de vários es$\operatorname{tudos}^{30}$. É nesse contexto que, na prática assistencial, destaca-se o trabalho da Fisioterapia na promoção de saúde como suporte aos familiares de pacientes com alta dependência. A conduta fisioterapêutica é proposta após a avaliação em domicílio abrangendo, igualmente, a figura do cuidador, objetivando a prevenção ou minimização da sobrecarga e o impacto emocional negativo gerado com a tarefa do cuidar.

Interação com os Agentes Comunitários de Saúde

- $\quad$ Considerando a grande demanda pela dificuldade de acesso aos serviços e a atuação do fisioterapeuta por meio do apoio matricial, a interação com os Agentes Comunitários de Saúde (ACS) é fundamental na realização de ações de acompanhamento às orientações dadas ao usuário e sua 
referentes aos cuidados com a saúde. A atuação, assim compreendida, pressupõe o envolvimento de toda a equipe de saúde, além da família, dos vizinhos e das lideranças comunitárias. No contexto das pessoas com deficiência, de classes populares, na condição de isolamento social em que vivem, a reabilitação com acompanhamento contínuo dos ACS potencializado pelo vínculo permite que o cuidado à saúde seja compartilhado continuadamente, auxiliando na construção de novas redes de apoio social e no empoderamento mútuo ${ }^{31}$. Pode-se perceber que o leque de intervenções é bastante extenso, porém, como apoiador matricial, a atuação do fisioterapeuta não deve estar descolada das demais profissões, pois se espera que haja planejamento prévio e de decisões em conjunto. As ações a serem realizadas devem estar direcionadas para as necessidades individuais e coletivas dos usuários da área em que a unidade de saúde está alocada. Conhecer o território, os aspectos epidemiológicos, as necessidades de saúde e a construção de planos de cuidado com a equipe de referência e com os outros membros da equipe de apoio matricial é basilar para que as ações desenvolvidas tenham identidade com os aspectos de saúde de cada comunidade. Outro ponto importante é a correlação com os outros níveis da atenção e a prática intersetorial. A APS não se constitui a única via na linha de cuidado, assim, torna-se importante a articulação com outros serviços ou arsenais terapêuticos ao longo de todo o processo para que se busque a integralidade do cuidado prestado.

\section{CONSIDERAÇÕES FINAIS}

A Fisioterapia vem-se tornando uma profissão cada vez mais procurada e utilizada, à medida que se redefinem seus horizontes de intervenção e ocorre um aumento na demanda social por suas ações e serviços ${ }^{33}$. O atendimento restrito na Atenção Secundária vem ocasionando uma demanda reprimida nesses locais. Além disso, há outro agravante ao acesso que é a dificuldade que grande parte dos usuários tem de se deslocar para esses serviços, tanto em função das limitações físicas como das questões econômicas (uma vez que esse atendimento pressupõe diversas idas ao serviço) $)^{31}$.

$\mathrm{Na}$ APS, que se revigora com a perspectiva da interdisciplinaridade, da integralidade do cuidado e da ação matricial, a demanda por atenção fisioterapêutica já começa a ser evidenciada e estimulada. Isso vem-se revelando intrinsecamente com a expansão da ESF, que vem colocando a assistência à saúde mais próxima da população e difundindo sua natureza essencialmente interdisciplinar. Nesse contexto, a inclusão do fisioterapeuta nas equipes de saúde aproxima e promove o acesso das pessoas a uma prática e a um saber que poderia continuar inatingível pelas dificuldades existentes de acesso e de exclusão.

Ao mesmo tempo, existe uma pressão dos profissionais dessas equipes no sentido de que seja disponibilizado atendimento fisioterapêutico para as pessoas acamadas e com sequela de AVE, que costumam ser a principal clientela encaminhada à Fisioterapia nesses serviços. Quando esse atendimento é disponibiliza- 
do, os profissionais de referência passam, então, toda a responsabilidade de recuperação daquele paciente crônico para o fisioterapeuta, de forma que esse profissional enquanto apoiador acaba se tornando o profissional de referência.

A inserção do fisioterapeuta, como também de outros profissionais, além da equipe mínima, não garante por si só que o cuidado se torne integral ou que não sofra soluções de continuidade. Por isso, tão fundamental quanto a sua inclusão na equipe, é estimular e garantir que a referência e o apoio realizem interconsultas, discussões de casos e a construção de planos de cuidado interdisciplinares segundo necessidades individuais, familiares e/ou comunitárias em busca de ações mais compartilhadas e efetivas. Devemos buscar que a inserção, nas práticas de atenção primária, de novas profissões e ações possa ser construída de forma a superar paradigmas históricos dessas profissões de predominante matriz biologicista. Outrossim, finalmente, que deva representar a integração de iniciativas de atores institucionais diversos. Abre-se, nesse contexto, e sob esse desafio ético-político, um grande oceano de possibilidades.

\section{REFERÊNCIAS}

1. Aciole GG. A Saúde no Brasil: cartografias do público e do privado. São Paulo: Hucitec; Campinas: Sindimed 2006.

2. Gil CRR. Atenção primária, atenção básica e saúde da família: sinergias e singularidades do contexto brasileiro. Cad Saúde Pública 2006; 22(6):117181.
3. De Lavor A, Domingues BC, Machado K, Carvalho M. A dívida com a Atenção Básica. Radis 2007; 59:12-17.

4. Escorel S, Giovanella L, MendonçaI MHM, Senna MCM. O Programa de Saúde da Família e a construção de um novo modelo para a atenção básica no Brasil. Rev Panam Salud Publica 2007; 21(2):164-76.

5. Rezende M. Avaliação da inserção do Fisioterapeuta na Saúde da Família de Macaé/RJ: a contribuição desde profissional para a acessibilidade da população idosa as ações de saúde das equipes: um estudo de caso [dissertação]. Rio de Janeiro (RJ): Escola Nacional de Saúde Pública Sérgio Arouca/Fiocruz; 2007.

6. Bispo Júnior JP. Fisioterapia e Saúde Coletiva: desafios e novas responsabilidades profissionais. Ciênc Saúde Coletiva 2010; 15(Supl. 1):1627-1636.

7. Brasil. Ministério da Saúde. Núcleos de Saúde Integral (versão 14). Brasília: MS; 2005.

8. Brasil. Ministério da Saúde. Portaria GM no 154, de 24 de janeiro de 2008. Cria os Núcleos de Apoio à Saúde da Família - NASF. Diário Oficial da União 2008; 4 mar.

9. Campos GWS, Domitti AN. Apoio matricial e equipe de referência: uma metodologia para gestão do trabalho interdisciplinar em saúde. Cad Saúde Pública 2007; 23(2):399-407.

10. Moura AAG, Carvalho EF, Silva NJC. et al. Repercussão das doenças crônicas não-transmissíveis na concessão de benefícios pela previdência social. Ciênc Saúde Coletiva 2007; 12(6):1661-72.

11. Brasil. Ministério da Saúde. Pactos pela Vida, em Defesa do SUS e de Gestão. Série pela saúde. Brasília (DF); 2006. 
12. Muniz CF, Arnaut AC, Yoshida M, Trelha CS, Dellaroza MSG. Projeto de assistência interdisciplinar ao idoso em nível primário: enfoque dos alunos de fisioterapia. Revista APS [internet] 2007 [acessado 2017 dez 11]; 10(1):84-89. Disponível em: http://www.uff.br/nates/files/2009/12/Pidoso.pdf

13. Mata MS, Costa FA, Souza TO, Mata ÁNS, Pontes JF. Dor e funcionalidade na atenção básica à saúde. Ciência \& Saúde Coletiva [internet] 2011 [acessado 2018 jan 11]; 16(1):221230. Disponível em: https://www. scielo.br/pdf/csc/v16n1/v16n1a25. pdf

14. Fonseca NR, Penna AFG. Perfil do cuidador familiar do paciente com sequela de acidente vascular encefálico. Ciênc Saúde Coletiva 2008; 13(4):1175-80.

15. Castro SS. Prevalência de deficiências e estado de saúde dos deficientes: Inquéritos de Saúde de base populacional realizado em municípios do Estado de São Paulo [dissertação]. São Paulo (SP): Faculdade de Saúde Pública da USP; 2006.

16. Brasil. IBGE [internet]. Ministério do Planejamento, Orçamento e Gestão. [acessado 2017 ago 13]. Disponível em: http://www.ibge.gov.br/home/ estatistica/populacao/censo2000/ tabulacao_avancada/tabela_gr_ uf_2.1.3.shtm

17. Neves LT, Aciole GG. Desafios da integralidade: Revisitando as concepções sobre o papel do fisioterapeuta na Estratégia de Saúde da Família. Interface (Botucatu)2011; 15(37); 551-564.
18. Almeida AB, Oliveira AMB, Ribeiro KSQS. A Fisioterapia na Atenção Básica a partir de uma experiência de Educação Popular. In: Anais do V Colóquio Internacional Paulo Freire; 2005; Recife, BR [internet]. Recife: UFPe; 2005 [acessado 2017 ago 24]. Disponível em: http://www. paulofreire.org.br/pdf/comunicacoes_orais/A FISIOTERAPIA NA ATENÇÃO BÁSICA A PARTIR DE UMA EXPERIÊNCIA DE EDUCAÇÃO POPULAR.pdf

19. Cardoso K. O fisioterapeuta como agente transformador na atenção básica [periódico na internet]. 2007 [acessado 2018 jan 5]. Disponível em: http://www.wgate.com.br/conteudo/medicinaesaude/fisioterapia/ variedades/agente_karina.htm

20. Silva DJ, Ros M. Inserção de profissionais de fisioterapia na equipe de saúde da família e Sistema Único de Saúde: desafios na formação. Ciência Saúde Coletiva 2007; 12(6):1673-81.

21. Ragasson CAP, Almeida DCS, Comparin K, Mischiati MF, Gomes JT. Atribuições do Fisioterapeuta no PSF: Reflexões a partir da Prática Profissional [monografia] [internet]. Cascavel (PR): Unioeste 2006 [acessado 2018 abr 10]. Disponível em: http://www.unioeste.br/projetos/ saudefamilia/atribuições_psf.rtf.

22. Gallo DLL. A fisioterapia no programa saúde da família: percepções em relação à atuação profissional e formação universitária [dissertação]. Londrina (PR): UEL; 2005. 
23. Sampaio RF. Promoção de saúde, prevenção de doenças e incapacidades: a experiência da Fisioterapia/UFMG em uma Unidade Básica de Saúde. Fisioter Mov 2002, 15(1):19-23.

24. Trelha CS, Silva DW, Iida LM, Fortes $\mathrm{MH}$, Mendes TS. O Fisioterapeuta no Programa de Saúde da Família em Londrina (PR). Espaç saúde 2007; 8(2):20-25.

25. Rezende M, Moreira MR, Amancio Filho A, Tavares MFL. A equipe multiprofissional da 'Saúde da Família’: uma reflexão sobre o papel do fisioterapeuta. Ciênc Saúde Coletiva 2009; 14 (Supl. 1):1403-1410, 2009.

26. Custódio LC, Oliveira BWA, Machado Neto CD, Moraes FAP, Moura RAA. Contribuições da fisioterapia para a promoção de saúde do cuidador informal. Revista APS 2007 [acessado $2018 \mathrm{fev} 12$ ]; 10(1):81-83. Disponível em: http://www.uff.br/ nates/files/2009/12/Cfisoterapia.pdf

27. Karsch U. Idosos dependentes: famílias e cuidadores. Cad Saude Publica 2003; 19(3):861-66.

28. Aveiro MC, AcioleI GG, Driusso P, Oishi J. Perspectivas da participação do fisioterapeuta no Programa Saúde da Família na atenção à saúde do idoso. Ciênc Saúde Coletiva 2011; 16(sup. 1):3129-3140, 2011.
29. Guedes TAL, et al. Metodologia e Atividades Desenvolvidas. In: Ribeiro KSQS, Lacerda DAL, organizadores. Fisioterapia na Comunidade: Experiência na Atenção Básica. João Pessoa: Edufpb; 2006. p. 115-71.

30. Cardia MCG. Manual da Escola de Posturas. $3^{\mathrm{a}}$ ed. João Pessoa: Editora Universitária UFPB; 2006.

31. Johnson J. Fisioterapia para o idoso. In: Kauffman TL, organizador. Manual de reabilitação geriátrica. Rio de Janeiro: Guanabara-Koogan; 2001. p. 366-69.

32. Ribeiro KS. et al. A Fisioterapia na Atenção Básica. In: Ribeiro KSQS, Lacerda DAL, organizadores. Fisioterapia na Comunidade: Experiência na Atenção Básica. João Pessoa: Editora Universitária UFPB; 2006. p. 67-79.

33. Castro AP, Neves VR, Aciole GG. Diferenças regionais e custos dos procedimentos de fisioterapia no Brasil, 1995 a 2008. Rev. Pan Saúde Pública, 30(5); 469-476:2011. 